\title{
Nonlinear phenomena in organic multilayers
}

\author{
V. M. Agranovich ${ }^{a}$, G. C. La Rocca ${ }^{b}$, F. Bassani ${ }^{b}$ and P. Reineker ${ }^{c}$ \\ ${ }^{a}$ Russian Academy of Sciences, Troitsk, Moscow obl., 142092 Russia \\ ${ }^{b}$ Scuola Normale Superiore and INFM, Piazza dei Cavalieri \\ I-56126 Pisa, Italy \\ 'Abteilung Theoretische Physik, Universität Ulm, 89069 Ulm, Germany
}

\begin{abstract}
In the last years there has been a surge of interest in the study of layered systems grown by organic molecular beam deposition. Such a progress has prompted us to consider novel layered structures containing either heterojunctions between a molecular solid and an inorganic semiconductor or donoracceptor interfaces between crystalline organic materials. Here, we review the striking resonant and non-resonant optical nonlinearities and electro-optical effects associated with the hybrid excitons and the interface charge transfer excitons characteristic of such systems.
\end{abstract}

\section{INTRODUCTION}

For many years there have been attempts to prepare molecular multilayer structures analogous to inorganic superlattice and quantum well structures. Recently, such structures have been prepared by molecular beam deposition methods and are undergoing intense studies by various groups in USA, Japan and Germany [1]. These molecular structures are held together by weak van der Waals forces rather than the valence or ionic forces, giving greater freedom and flexibility in preparing structures which may even be incommensurate. Recent progress in molecular beam deposition techniques led to the preparation of organic multilayered structures of high optical quality. In contrast to bulk materials one can tailor these nanoscale structures in order to change physical parameters of interest. These novel engineered materials open up a new field of research, which is very promising from the technological as well as the scientific point of view. However, experimental investigations of this new type of nanostructures are still at an early stage.

Until recently, the theoretical investigations carried out were not directly connected with the experimental studies. Nevertheless, it was recognized and demonstrated that the different interactions taking place at the interfaces are of great importance and can be responsible for the appearance of new linear and nonlinear optical effects. Thus, the recent theoretical analysis of the gas-condensed matter shift of energy levels of molecules near the interface showed that in such crystalline layered molecular structures the flow of energy can be controlled, and sometimes electronic excitations can be concentrated near interfaces between different layers [2]. It was shown also that different types of Fermi resonances can be used as a universal mean for achieving optical bistability $[2,3]$ and multistability [2, 4]. Also time dependent periodic and non-periodic solutions were obtained [5]. New states, Fermi resonance interface modes [6] and Fermi resonance interface solitons [7, 8], were predicted. The existence of these states may give rise to a resonant enhancement of the quadratic and cubic nonlinear optical susceptibilities [9], and bistability or even multistability of energy transmission across the interface $[3,4]$. It was found that electronic states at the interface between organic and inorganic materials also can be potentially interesting. It was shown that in coupled organic-inorganic quantum wells a novel type of electronic excitations can exist, which are due to the hybridization of Frenkel and Wannier exciton states [10]. The same phenomenon for organic and semiconductor quantum wires has also been considered [11]. These new excitons give rise to very pronounced optical nonlinearities 
because they have at the same time large oscillator strengths, typical of Frenkel exsitons, and large radii, typical of Wannier excitons. The results of calculations of the microscopic optical properties of such systems show that enhancements of two orders of magnitude both in the value of the susceptibility (linear part) and in its relative change with increasing excitation density (Kerr nonlinearity) should take place [12] (with respect to those measured in semiconductor quantum wells).

New and unusual optoelectronic properties are predicted in donor-acceptor (D-A) interfaces and organic superlattices. In such structures the lowest energy electronic excitations are charge-transfer excitons (CTEs) at a D-A interface. These excitons exhibit large static electric dipole moments (all oriented in the same direction, i.e., perpendicular to the interface) and experience the strongest (among other types of excitons) dynamical exciton-exciton interaction (exciton-exciton repulsion). Due to this interaction at high CTE concentrations many new and interesting effects occur. For instance, an insulator to metal transition [13] can take place, an electrostatic potential drop can provide a novel photovoltaic mechanism in asymmetrical D-A superlattices [14]. This interaction may also result in striking and unusual resonant and off-resonant NLO responses with peculiar excitation intensity dependences [15]. The theoretical investigations carried out so far accompanied with the modern level of technology lead us to believe that D-A structures are feasible as a novel class of materials for nonlinear optics. More specifically, this project is devoted to theoretical and experimental investigations of organic multilayered D-A nanostructures.

Here, we review our recent theoretical studies of the electronic and optical properties of such layered structures. In particular, we focus on the peculiar electro-optical and nonlinear optical properties of two-dimensional charge transfer excitons at donor-acceptor interfaces and on the large resonant optical nonlinearities of hybrid excitons in organic-inorganic heterostructures.

\section{CHARGE-TRANSFER EXCITON OPTICAL NONLINEARITIES}

The interaction energy between two CTE having a dipole moment $\mu$ (which is typically 20 Debye) at a distance $\rho$ along the D-A interface plane is $U=\mu^{2} /\left(\kappa \rho^{3}\right)$ where $\kappa$ is the static dielectric constant (typically, $\kappa \simeq 3$ ); for instance, for $\rho \simeq 5 \AA, U \simeq 1 \mathrm{eV}$. The average exciton-exciton distance $\rho_{\circ}$ is related to the two-dimensional (2D) density of CTEs $n$ by $n \simeq 1 / \rho_{0}^{2}$. The ensuing repulsion increases the energy of the CTEs and the corresponding energy shift $\Delta \epsilon$ is given by the average interaction of one exciton with all the others. We can expect that the CTEs having a non negligible mobility will tend to order in such a way as to minimize the energy of repulsion. Assuming for the sake of simplicity that the CTEs are located at the sites of a square lattice, we find $\Delta \epsilon \simeq 10 \mu^{2} /\left(\kappa \rho_{o}^{3}\right)=10 V_{o}(n / N)^{(3 / 2)}$, with $V_{o}=\mu^{2} /\left(\kappa a^{3}\right)$ and $\mathrm{N}$ the total $2 \mathrm{D}$ density of molecules given by $N=1 / a^{2}$ where $a$ is the molecular crystal lattice constant along the interface (which is of order $5 \AA$ ). This estimate is expected to be valid even for a disordered homogeneous distribution of CTEs of comparable $2 \mathrm{D}$ density $n$. We wish to stress here the non-analytic dependence of $\Delta \epsilon$ on $n$ : the leading correction scales like $n^{3 / 2}$. The D-A interface polarizability due the CTE contribuition can be written as $\chi(\omega) \simeq A /\left(\left(\epsilon_{0}+\Delta \epsilon\right)^{2}-\omega^{2}\right)$, where $\epsilon_{0}$ is the CTE energy for $n=0$ and $A$ is a constant proportional to the CTE oscillator strength. Expanding $\chi(\omega)$ in series of $\Delta \epsilon / \epsilon_{o}$ we find that $\chi(\omega)=\chi_{o}^{(1)}(\omega)\left(1-2 \epsilon_{0} \Delta \epsilon(n) /\left(\epsilon_{o}^{2}-\omega^{2}\right)\right)=\chi_{o}^{(1)}+\Delta \chi(n)$ where $\chi_{o}^{(1)}$ is the polarizability for $n=0$. Thus, for the nonlinear correction to the polarizability corresponding usually to the Kerr nonlinearity, we have $\Delta \chi / \chi_{0}^{(1)}=-2 \epsilon_{0} \Delta \epsilon /\left(\epsilon_{o}^{2}-\omega^{2}\right) \simeq-\Delta \epsilon /\left(\epsilon_{o}-\omega\right)$, where for resonant pumping $\left|\epsilon_{o}-\omega\right| \simeq \delta, \delta$ being the exciton linewidth. Assuming the concentration $n \simeq N / 100$ and using the previous estimates, we have $\Delta \epsilon \simeq 50 \mathrm{~cm}^{-1}$ which, even for $\delta \simeq 1000 \mathrm{~cm}^{-1}$ gives for the resonant nonlinearity $\Delta \chi / \chi_{0}^{(1)} \simeq 0.05$. Such change in polarizability is not due to the twolevel-system-like anharmonicity which is the main nonlinear mechanism for Wannier-Mott excitons in semiconductors (phase space filling [16]), but is caused by the dynamical exciton-exciton interaction. In fact, we have that the figure of merit $\Delta \chi / \chi_{0}^{(1)}$ is almost one order of magnitude larger than $n / n_{S}$ where the CTE saturation density $n_{S}$ is given by $N$ itself. A 2D concentration along each interface of 0.01 can hardly be dangerous for organic crystals as, taking into account the thickness of the layer between successive interfaces, it may correspond to a 3D concentration of electronic excitation of order $10^{-3}$. The continuous wave (CW) optical nonlinearity of $2 \mathrm{D}$ CTEs here considered goes 
beyond the usual perturbation theory $\dot{a}$ la Bloembergen. As a matter of fact, because in steady state equilibrium conditions $n \propto I \propto|E(\omega)|^{2}$ ( $I$ being the pump light intensity), the shift of the CTE energy $\Delta \epsilon(n)$ and the nonlinear correction to the polarizability turn out to be proportional to $|E|^{3}$. Thus, the nonlinear part of the polarization is $\Delta \vec{P}(\omega) \propto|E(\omega)|^{3} \vec{E}(\omega)$ and such a term can not be found in the usual expansion of the polarization $\vec{P}$ in powers of the components of the electric field $\vec{E}$. This peculiarity stems from the long-range exciton-exciton interaction that shifts the CTE energy in a non-analytic way with respect to $n$. The unusual dependence of $\Delta \vec{P}$ on $\vec{E}$ here predicted should be easily experimentally observed for $\mathrm{CW}$ resonant pumping. We recall that the usual Kerr nonlinearity contribute to the nonlinear polarization $\Delta \vec{P}(\omega) \propto|E(\omega)|^{2} \vec{E}(\omega)$, so that the corresponding correction to the susceptibility is proportional to the field intensity $I(\omega)$. In the case considered this correction is proportional to $I(\omega)^{3 / 2}$ and the task of the experiment is to reveal this stronger dependence.

It is already clear from all of the above that the most suitable D-A structures to observe the effects described above are D-A multilayered structures in which: $i$ ) the CTEs are the lowest energy electronic excitations, ii) in the vicinity of the CTE resonance the main contribution to the susceptibility should come from the CTE resonance alone and iii) the width of CTE resonance should be as small as possible. The effect described above may be observed not only at the interfaces of two crystalline materials but also at the interface between a polymer and a crystalline material (e.g., a polymer as a donor, $\mathrm{C}_{60}$ as an acceptor).

\section{PHOTOGENERATED STATIC ELECTRIC FIELD}

In the above discussion, we have considered the effects due to the CTE-CTE repulsion, which contribute to the resonant nonlinear absorption (as well as to other resonant nonlinearities) by the CTEs themselves. A more general mechanism by which the nonlinear optical properties of media containing CTEs can be affected is due to the strong static electric field arising in the vicinity of an excited CTE. If, for example, the CTE (or CT complex) static electric dipole moment is 20 Debye, at a distance of $5 \AA$ it creates a field $E^{C T E}$ of order $10^{7} \mathrm{~V} / \mathrm{cm}$. Such strong electric fields change the hyperpolarizabilities $\alpha, \beta, \gamma$, etc., of all molecules close to the CTE. For instance, in the presence of this CTE induced static fields, the microscopic molecular hyperpolarizabilities are modified as follows $\alpha_{i j}=\alpha_{i j}^{(0)}+\alpha_{i j l}^{(1)} E_{l}^{C T E}+\alpha_{i j l k}^{(2)} E_{l}^{C T E} E_{k}^{C T E}+\ldots, \beta_{i j l}=\beta_{i j l}^{(o)}+\beta_{i j l k}^{(1)} E_{k}^{C T E}+\ldots$, etc.. The changes in the molecular hyperpolarizabilities are reflected in all the macroscopic nonlinear optical constants of a medium. As the CTE induced electrical fields increase with the concentration of CTEs, a stronger dependence of the nonlinear optical response on the intensity of light is expected. For example, $\chi^{(3)}$ will include a contribution from $\chi^{(4)}$ and the corresponding polarization will be given by $\Delta P_{i} \simeq \chi_{i j l m}^{(3)} E_{j} E_{l} E_{m}+\chi_{i j l m q}^{(4)} E_{j} E_{l} E_{m} E_{q}^{C T E}$ where $E$ is the pump light electric field, therefore, if the second term is not negligible, $|\Delta P|^{2}$ will depend on the pump intensity $I \propto|E(\omega)|^{2}$ more strongly than like $I^{3}$, through the dependence of $E^{C T E}$ on $I$. The macroscopic susceptibilities will depend on an average over the positions and orientations of the static electric dipoles of the CTEs photogenerated in the medium. The influence of static electric fields on the molecular hyperpolarizabilities has long been known, but in our case the static field effects are controlled by the pumping light as they are associated to the presence of the CTEs: this can lead to a novel class of all optical nonlinearities. We recall that the considered influence of the CTEs can occur only if the CTEs are the lowest energy long-living electronic excitations.

The same idea also applies to noncrystalline polymeric media with impurities having a CT complex in the excited state. If the polymers are approximately oriented in one direction and we have a more or less ordered 1D system of CTEs having a strong repulsive interaction we can expect again, as for 2D CTEs, a large enhancement of the optical nonlinearities and a stronger intensity dependence of the nonlinear optical response. Indeed, for an ordered 1D system the correction to the energy is proportional to $n^{3}\left(\Delta \epsilon \propto \mu^{2} / \rho_{o}^{3}, \rho_{o} \simeq 1 / n, n\right.$ being the $1 \mathrm{D}$ density) and the nonlinear contribution to the polarizability due to the CTE-CTE repulsion is proportional to $|E(\omega)|^{6}$. Such a contribution corresponds to a nonlinear polarization $\Delta \vec{P}(\omega) \propto|E(\omega)|^{6} \vec{E}(\omega)$. It means that the appearance of 
CTEs under the influence of a strong illumination can change drastically the intensity dependence of the nonlinear optical properties and just this theoretical prediction could be checked easily by experiments. The CTE contribution to the nonlinear absorption coefficient is proportional to $I^{3 / 2}$ for the $2 \mathrm{D}$ case and to $I^{3}$ for the $1 \mathrm{D}$ case, instead of the usual linear dependence on $I$. Noncrystalline polymeric media with impurities having a CT complex in the excited state are not an ordered $1 \mathrm{D}$ case. In this case the intensity dependence depends drastically on the structure of polymers. The corresponding effects for polymers are not investigated theoretically up to now.

\section{RESONANT HYBRID EXCITON OPTICAL NONLINEARITIES}

In a system containing two quantum wells, one of an inorganic semiconductor and the other of a molecular solid, a new type of electronic excitations due to the hybridization of Frenkel and Wannier exciton states has been considered [10,12]. The physical system of interest comprises two parallel two-dimensional (2D) layers separated by a distance $z_{o}$ : the first sustains tightly bound Frenkel excitons (FE) and the second loosely bound Wannier excitons (WE) having (nearly) the same energy. These resonating electronic excitations have the same center of mass momentum $\vec{Q}$ along the plane of the layers (assuming a perfect bidimensional translational symmetry). They can interact with light having a parallel component of its wavevector equal to $\vec{Q}$ and an energy close to that of the excitons. Structures with inorganic and/or organic well thicknesses comparable to $z_{o}$ can similarly be considered, in which case a realistic description of quasi-two-dimensional excitons should be used. Even though the first and second layers are referred to as the organic and inorganic (semiconductor) quantum well, respectively, this model applies to any heterostructure with layers sustaining small radius and large radius resonant excitons. Due to the dipole-dipole interaction between the $\mathrm{FE}$ and the WE, the true excitations of the system are hybrid excitons (HEs). When the FE-WE mixing is (nearly) complete, the 2D HEs are expected to exhibit strong optical nonlinearities in view of the large oscillator strength of the small radius excitons and the small saturation density of the large radius excitons $[12,17]$.

The calculation of the (nonlinear) optical susceptibility, starting with a microscopic Hamiltonian expressed in terms of fermionic operators, leads to the derivation of the interband polarization driven by the light field, along the lines of the standard formalism used for semiconductor structures $[17,18]$. The total Hamiltonian $H$, besides the usual terms describing the electrons and holes in the organic and inorganic well, respectively, and their coupling to the external field, contains the Coulomb interaction terms between the carriers in one layer and those in the other. This interlayer coupling has two contributions: one is an intraband interaction that does not change the number of electrons and holes in either layer, the other is an interband interaction corresponding to the destruction of an electronhole pair in one layer and the creation of a pair in the other. Usually, the Coulomb interband scattering can be neglected, but not in this case because of the resonance condition between the exciton in one layer and that in the other. The intraband term of the interlayer coupling does not contribute to the equation of motion of the interband polarization and is here of no interest; the interband interaction (stemming from the electron-hole exchange) is responsible for the FE-WE hybridization and the novel nonlinearities which are the focus of this paper. This term of the Hamiltonian can be written as

$$
H_{\text {hyb }}=\sum_{\vec{k}, \overrightarrow{k^{\prime}}, \vec{q}}\left(V_{\text {hyb }}(\vec{q}) c_{\vec{k}+\vec{q}}^{\dagger} h_{-\vec{k}}^{\dagger} m_{-\overrightarrow{k^{\prime}}+\vec{q}} u_{\overrightarrow{k^{\prime}}}+\text { h.c. }\right)
$$

where $c(u)$ and $h(m)$ are, respectively, the electron and hole operators in the semiconductor (organic) layer, and $\vec{k}, \overrightarrow{k^{\prime}}, \vec{q}$ are two-dimensional wavevectors parallel to the layers. A straightforward calculation gives

$$
V_{\mathrm{hyb}}(\vec{q})=\frac{2 \pi q e^{-z_{o} q}}{\epsilon A}\left(d_{c h \|} d_{u m \|}^{*}-d_{c h z} d_{u m z}^{*}+i d_{c h \|} d_{u m z}^{*}+i d_{c h z} d_{u m \|}^{*}\right)
$$


where $A$ is the normalization area along the layers, $\vec{d}_{c h}\left(\vec{d}_{u m}\right)$ the transition electric dipole in the inorganic (organic) layer, and $\|$ refers to the component along $\vec{q}$.

From the interband polarization functions in the inorganic $\left(P_{W}(\vec{k})=<h_{-\vec{k}} c_{\vec{k}+\vec{Q}}>\right)$ and organic $\left(P_{F}(\vec{k})=<m_{-\vec{k}} u_{\vec{k}+\vec{Q}}>\right.$ ) layers, the optical polarization per unit area $\vec{P}$ (driven by the light electric field $\vec{E})$ and the corresponding susceptibility $\chi$ are obtained as follows

$$
\vec{P}=\frac{2}{A} \sum_{\vec{k}}\left(\vec{d}_{u m}^{*} P_{F}(\vec{k})+\vec{d}_{c h}^{*} P_{W}(\vec{k})\right)=\chi \vec{E}
$$

The interband polarization functions are calculated solving their coupled equations of motion (semiconductor Bloch equations) with the usual approximations[17, 18]; in particular, the light-matter interaction is described semiclassically in the rotating wave approximation, the Coulomb interaction is treated at the unscreened Hartree-Fock level, only the lowest exciton state in each layer is considered and the nonlinearities are included in first order perturbation theory in the total exciton density $N$. Neglecting the Wannier exciton oscillator strength $\left(F_{O r} \geq 100 F_{I n}\right)$ and the Frenkel exciton nonlinearities (their saturation density is more than 100 times larger than that of WEs), the final result has the following form

$$
\chi(\omega ; N) \simeq \frac{F_{O r}\left(E_{I n}^{0}+E_{I n}^{1}-\hbar \omega\right)}{\left(E_{I n}^{0}+E_{I n}^{1}-\hbar \omega\right)\left(E_{O r}^{0}-\hbar \omega\right)-B_{I n}\left|V_{W F}^{0}+V_{W F}^{1}\right|^{2}}
$$

where the nonlinearities enter through the Pauli blocking factor: $B_{I n} \simeq 1-0.14 \pi a_{B}^{2} N$, the WE blue shift: $E_{I n}^{1} \simeq 0.48 E_{b} \pi a_{B}^{2} N$ (a $a_{B}$ being the Bohr radius of the Wannier exciton and $E_{b}$ its binding energy), the renormalized hybridization: $\left|V_{W F}^{0}+V_{W F}^{1}\right|^{2} \simeq\left(1-0.12 \pi a_{B}^{2} N\right)\left|V_{W F}^{0}\right|^{2}$. While the saturation density is comparable to that of Wannier excitons $\left(n_{S} \approx 1 / a_{B}^{2}\right)$, the density of photogenerated electron-hole pairs, for a given light intensity, can be two orders of magnitude larger $\left(F_{O r} / F_{I n} \approx a_{B}^{2} / r_{0}^{2} \approx 100\right.$ ); for the same reason, also the linear susceptibility $\chi_{0}$ can be two orders of magnitude larger. Therefore, the present theory substantiates the intuitive expectation of very pronounced nonlinear optical properties of the hybrid excitons.

Many different experimental arrangements are feasible to study the nonlinear optics of hybrid excitons; in particular, a coupling grating configuration would allow to excite excitons with a wavevector $\vec{Q}_{M}$ corresponding to the maximum of $\left|V_{W F}^{0}\right|[12]$. Other experimental techniques typical of surface excitation studies such as attenuated total reflection measurements could also be employed. For a numerical estimate, we use the following set of parameters: $z_{0} \simeq 30 \AA, r_{0} \simeq 5 \AA$ (radius of the FE, assumed to be very tightly bound), $a_{B} \simeq 80 \AA, E_{b} \simeq 10 \mathrm{meV}, d_{u m} \simeq 11$ Debye, $d_{c h} \simeq 12$ Debye, $\epsilon \simeq 9$ (background dielectric constant); which gives $\left|V_{W F}^{0}\right| \simeq 4 \mathrm{meV}$ at $Q \simeq 2 \cdot 10^{6} \mathrm{~cm}^{-1}$. Fig. 1 shows the uncoupled WE and FE dispersions together with the upper and lower HE dispersions, the $Q$ dependent coupling interaction is also illustrated. Fig. 2 shows the two-dimensional susceptibility near the split resonance of the hybrid excitons at low, medium and high excitation densities; it is noticeable, in particular, that in the first case (linear regime) the mixing is complete and the oscillator strength is equally shared by the two peaks, in the other cases (nonlinear regime), due to the small blue shift of the WE, the stronger line corresponds to the lowest (more Frenkel-like) hybrid exciton. Assuming to have a multiple double-well structure with a period of about $600 \AA$ (for which the double-well units are decoupled from one another) and including a phenomenological linewidth $\hbar \gamma$ of $2 \mathrm{meV}$ for both the organic and inorganic excitons (as in Fig. 2), we obtain for the fractional nonlinear change in absorption coefficient close to resonance $|\Delta \alpha| / \alpha \simeq 3 \cdot 10^{-12} \mathrm{~cm}^{2} N_{T}$, which is of the same order as for a semiconductor multiple quantum well, but for a given pump intensity $I_{P}$ the $2 \mathrm{D}$ density of photogenerated excitons $N_{T}$ is in our case about two orders of magnitude larger 


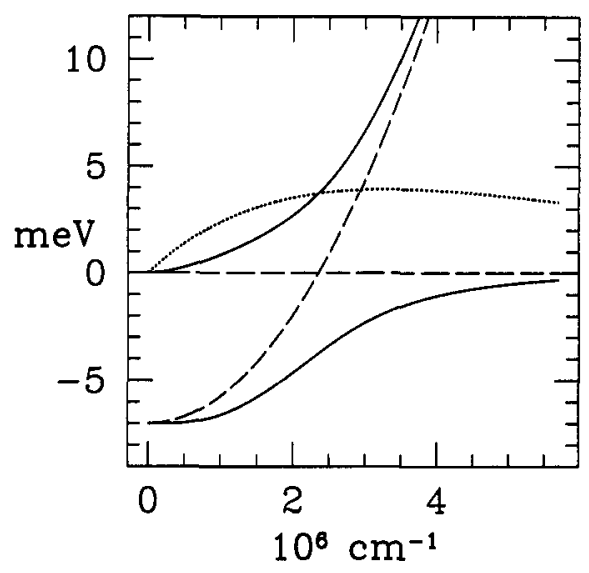

Figure 1: Dispersion of the uncoupled FE and WE (long-dashed lines) and of the upper and lower HEs (solid lines), the energy scale being centered at the FE energy; also shown is the $Q$ dependent coupling $\left|V_{W F}^{0}\right|$ (dotted line).

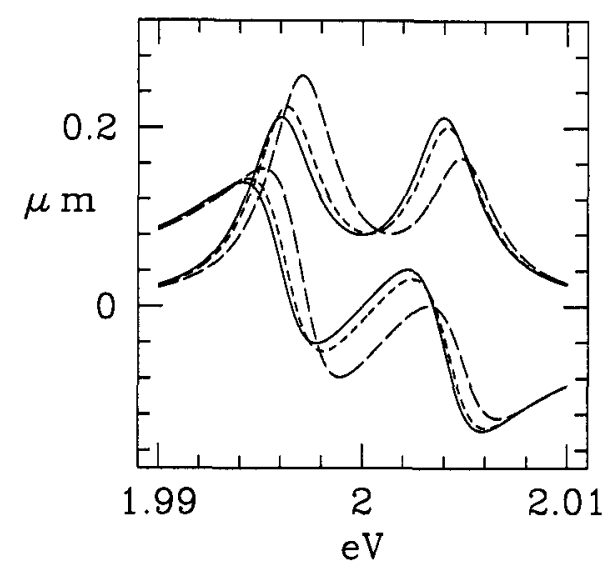

Figure 2: Real and imaginary parts of the two-dimensional susceptibility $\chi$ near the hybrid exciton doublet: in the linear regime (solid lines), at medium excitation density $\left(n_{T} \simeq 5 \cdot 10^{10} \mathrm{~cm}^{-2}\right.$, short-dashed lines) and at high excitation density $\left(n_{T} \simeq 2 \cdot 10^{11} \mathrm{~cm}^{-2}\right.$, long-dashed lines), see text for details.

because the oscillator strength of hybrid excitons is comparable to that of Frenkel excitons rather than Wannier excitons $\left(N_{T} \propto I_{P} F_{O_{r}}\right)$.

Regarding the practical implementation of the physical system here considered, examples of molecular substances having small radius $(\leq 10 \AA)$ excitons with energies of a few eV, among those already successfully grown [1] by OMBD as crystalline layers on a variety of inorganic (including semiconductor) crystals, are aromatic hydrocarbons, such as tetracene $(2 \mathrm{eV})$ or pentacene $(1.5 \mathrm{eV})$, metallophtalocyanines, such as VOPc $(1.6 \mathrm{eV})$ or $\mathrm{CuPc}(1.8 \mathrm{eV})$, and tetracarboxilic compounds, such as NTCDA $(3.1 \mathrm{eV})$ or PTCDA $(2.2 \mathrm{eV})$. Semiconductors having large radius excitons with matching energies are, for instance, the III-V and II-VI ternary solid solutions such as GaAlAs, ZnCdSe and ZnSSe [19]; beside a judicious choice of alloy composition and well thickness, a fine tuning of the resonance condition could be achieved applying an external static electric field along the growth direction (quantum confined Stark effect [17]). A major experimental problem is the control of the interface quality: the inhomogeneous broadening should remain small and the parallel wavevector $\vec{Q}$ a (sufficiently) good quantum number; organic superlattices with high quality interfaces have been demonstrated, for example, by Akimichi et al. [1]. An additional problem with many organic substances is the rather large homogeneous broadening due to a strong electron-phonon interaction, but there are also favorable cases, such as for the surface $2 \mathrm{D}$ exciton in anthracene $(3.1 \mathrm{eV})$ which has a linewidth of the order of $1 \mathrm{meV}$ [20]. In principle, though, apart from the resonance condition and large difference in excitonic radii, the present model demands no specific requisite and the rapid development of OMBD [1] justifies some optimism about its concrete realization.

In summary, the nonlinear optical properties of novel hybrid excitons in organic-inorganic heterostructures have been calculated within a microscopic model. With respect to the usual semiconductor quantum wells, a very strong enhancement of both the linear and nonlinear parts of the susceptibility is predicted. These results are rationalized in terms of the large oscillator strength of Frenkel excitons and the low saturation density of Wannier excitons: both characteristics at the same time belong to the hybrid resonances. If succesfully synthesized, structures of the type here considered would exhibit pronounced nonlinearities of potential technological interest. Finally, the hybrid excitons are 
a promising system for novel electro-optical and magneto-optical properties.

\section{CONCLUSIONS}

The method of OMBD combined with a careful substrate preparation and sample characterization has been successful in growing layered systems including organic crystalline materials (e.g., tetracene, perylene, fullerene, PTCDA). There is little doubt that in the future the number of molecular species used will rapidly increase and that a large flexibility in the design of novel type of structures will be achieved. In such systems, we can expect the appearence of unusual properties in a variety of situations. Here, we have theoretically studied organic-inorganic heterostructures sustaining a new kind of excitons (i.e., hybrid excitons) which have large optical nonlinearities, and also systems with donor-acceptor interfaces and two-dimensional charge transfer excitons giving rise to peculiar alloptical nonlinearities. We believe that this is a very promising field of research and hope that the experimental efforts to synthesize and investigate these novel structures will be successful.

\section{ACKNOWLEDGEMENT}

V. M. A. is grateful for the support and hopitality of the Scuola Normale Superiore (Pisa) and for partial support from the Russian Foundation of Basic Research (Grant 96-03344049). The support from the Volkswagen Foundation (V. M. A. and P. R.) is gratefully acknowledged.

\section{REFERENCES}

1. See for instance: F.F. So, S.R. Forrest, Y.Q. Shi and W.H. Steier, Appl. Phys. Lett. 56, 674 (1990); N. Karl and N. Sato, Mol. Cryst. Liq. Cryst. 218, 79 (1992); H. Akimichi, T. Inoshita, S. Hotta, H. Noge, and H. Sakaki, Appl. Phys. Lett. 63, 3158 (1993); Y. Imanishi, S. Hattori, A. Kakuta, and S. Numata, Phys. Rev. Lett. 71, 2098 (1993); T. Nonaka, Y. Mori, N. Nagai, Y. Nakagawa, M. Saeda, T. Takahagi and A. Ishitani, Thin Solid Films, 239, 214 (1994); E.I. Haskal, Z. Shen, P.E. Burrows and S. R. Forrest, Phys. Rev. B 51, 4449 (1995); E. Umbach, C. Seidel, J. Taborski, R. Li, and A. Soukopp, phys. stat. sol. (b) 192, 389 (1995)

2. V.M. Agranovich, Molec. Cryst. Liq. Cryst. 230, 13 (1993); Nonlinear Optics 9, 87 (1995); Physica Scripta T49, 699 (1993); V.M. Agranovich, R. Atanasov and F. Bassani, Chem. Phys. Lett. 199, 621 (1992).

3. V.M. Agranovich and J.B. Page, Phys. Lett. A183, 395 (1993).

4. J.B. Page and V.M. Agranovich, Bull. Am. Phys. Soc. 39, 594 (1994).

5. P. Reineker, Th. Neidlinger, V.M. Agranovich. and V.I. Yudson, J. Lumin. 66\&67, 453 (1996).

6. V.M. Agranovich and O.A. Dubovsky, Chem. Phys. Lett. 210, 458 (1993).

7. V.M. Agranovich and A.M. Kamchatnov, JETP Letters 59, 397 (1994).

8. V.M. Agranovich, S.A. Darmanyan, O.A. Dubovsky, A.M. Kamchatnov, E. Ogievetsky, Th. Neidlinger, and P. Reineker, Phys. Rev. B 53, 1545 (1996).

9. V.M. Agranovich, P. Reineker and V.I. Yudson, Synthetic Metals 64, 147 (1994); Th. Neidlinger, P. Reineker, V.M. Agranovich, and V.I. Yudson, Nonlin. Optics 9, 49 (1995).

10. V.M. Agranovich, R. Atanasov and F. Bassani, Sol. State Comm. 92, 295( 1994). 
11. V.I. Yudson, P. Reineker and V.M. Agranovich, Phys. Rev. B 52, R 5543 (1995); Nuovo Cimento D 17, 1797 (1995); P. Reineker, V.M. Agranovich, V.I. Yudson, Mol. Cryst. Liquif. Cryst. 283, 215 (1996).

12. G.C. La Rocca, F. Bassani and V.M. Agranovich, Il Nuovo Cimento D 17, 1555 (1995); G.C. La Rocca, Physica Scripta T (1996, in the press).

13. V.M. Agranovich and K.N. Ilinsky, Phys. Lett. A 191, 309 (1994).

14. V.M. Agranovich, G.C. La Rocca and F. Bassani, JETP Letters 62, 405 (1995).

15. V.M. Agranovich, G.C. La Rocca and F. Bassani, Chem. Phys. Lett. 247, 355 (1995). V.I. Yudson, Th. Neidlinger, and P. Reineker, Phys. Lett. A 204, 313 (1995); P. Reineker, Th. Neidlinger, and V.I. Yudson, J. Lumin. 66\&67, 453 (1996).

16. B.I. Green, J. Orenstein, S. Schmitt-Rink, Science 247, 679 (1990).

17. S. Schmitt-Rink, D. S. Chemla, D. A. B. Miller, Phys. Rev. B 32, 6601 (1985); S. Schmitt-Rink, D. S. Chemla, D. A. B. Miller, Advances in Physics 38, 89 (1989).

18. H. Haug and S. W. Koch, "Quantum Theory of the Optical and Electronic Properties of Semiconductors", 2nd ed. (1993), Singapore: World Scientific; R. Zimmermann, "Many Particle Theory of Highly Excited Semiconductors", Teubner Texte zur Physik 18 (1988), Leipzig: Teubner.

19. Landolt-Börnstein Vol. III/22a, Ed. O. Madelung, Springer-Verlag, Berlin (1987).

20. M. Orrit, J. Bernard, J. M. Turlet, Ph. Kottis, J. Chem. Phys. 78, 2847 (1983). 